FERMILAB-Conf-95/012-E

DØ

\title{
Cosmic Ray Test Results of the DØ Prototype Scintillating Fiber Tracker
}

\author{
D. Adams et al. \\ Fermi National Accelerator Laboratory \\ P.O. Box 500, Batavia, Illinois 60510
}

January 1995

To appear in the proceedings of the 4th International Conference on Advanced Technology and Particle Physics, Como, Italy, October 3-7, 1994 


\section{Disclaimer}

This report was prepared as an account of work sponsored by an agency of the United States Government. Neither the United States Government nor any agency thereof, nor any of their employees, makes any warranty, express or implied, or assumes any legal liability or responsibility for the accuracy, completeness, or usefulness of any information, apparatus, product, or process disclosed, or represents that its use would not infringe privately owned rights. Reference herein to any specific commercial product, process, or service by trade name, trademark, manufacturer, or otherwise, does not necessarily constitute or imply its endorsement, recommendation, or favoring by the United States Government or any agency thereof. The views and opinions of authors expressed herein do not necessarily state or reflect those of the United States Government or any agency thereof. 


\title{
Cosmic ray test results of the $\mathrm{D} \emptyset$ prototype scintillating fiber tracker*
}

\author{
D. Adams ${ }^{h}$, M. Adams ${ }^{b}$, B. Baumbaugh ${ }^{f}$, I. Bertram ${ }^{h}$, A. Bross ${ }^{a}$, D. Casey ${ }^{i}$, S. Chang ${ }^{e}$, M. Chung ${ }^{b}$, \\ C. Cooper ${ }^{g}$, C. Cretsinger ${ }^{i}$, R. Demina ${ }^{e}$, G. Fanourakis ${ }^{i}$, S. Grünendahl ${ }^{i}$, J. Hinson ${ }^{g}$, B. Howell ${ }^{g}$, \\ H. Joharie, J. S. Kang ${ }^{c}$, C. L. Kim ${ }^{c}$, S. K. Kim ${ }^{j}$, D. Koltick ${ }^{g}$, F. Lobkowicz ${ }^{i}$, S. Margulies ${ }^{b}$, \\ J. Moromisato ${ }^{e}$, M. Narain ${ }^{a}$, C. H. Park ${ }^{a}$, Y. M. Park ${ }^{d}$, S. Reucroft ${ }^{e}$, R. Ruchti ${ }^{f}$, J. Solomon $^{b}$, \\ E. VonGoeler ${ }^{e}, J$. Warchol ${ }^{f}$, M.Wayne ${ }^{f}$, E.Won ${ }^{i}$, and Y.Yu ${ }^{j}$ \\ ${ }^{a}$ Fermi National Accelerator Laboratory, Batavia, Illinois 60510 , \\ ${ }^{b}$ University of Illinois at Chicago, Chicago, Illinois 60680 , \\ ${ }^{c}$ Korea University, Seoul, Korea 136-701, \\ ${ }^{d}$ Kyungsung University, Pusan, Korea 608-736, \\ ${ }^{e}$ Northeastern University, Boston, Massachusetts 02115, \\ ${ }^{f}$ University of Notre Dame, Notre Dame, Indiana 46556, \\ ${ }^{g}$ Purdue University, Lafayette, Indiana 47907, \\ ${ }^{h}$ Rice University, Houston, Texas 77251, \\ ${ }^{i}$ University of Rochester, Rochester, New York 14627 , \\ ${ }^{j}$ Seoul National University, Scoul, Korea 151-742
}

The performance of a large scale scintillating fiber tracker with VLPC readout has been studied in a cosmic-ray test. Approximately 9.6 photoelectrons per single layer per trigger were detected at a VLPC bias voltage of $6.5 \mathrm{~V}$. The doublet efficiency was nearly $100 \%$ at a $0.1 \%$ noise level and a position resolution of about $140 \mu \mathrm{m}$ was measured. We also studied the relationship between VLPC performance and VLPC bias voltage by measuring single fiber efficiency as a function of VLPC bias in the range $6.2 \mathrm{~V}$ to $7.0 \mathrm{~V}$ at a fixed temperature of $6.5^{\circ} \mathrm{K}$. We observed no significant variation in VLPC performance within this bias range.

\section{INTRODUCTION}

The $\mathrm{D} \emptyset$ scintillating fiber tracker is one of the major components of the D $\emptyset$ upgrade project[1]. This upgrade will allow the $\mathbf{D} \emptyset$ detector to take full advantage of the physics opportunities that will be available at an upgraded Tevatron with increased luminosity. The fiber tracker will consist of approximately 80000 fibers arranged in four super layers. The fiber tracker, used together with a new silicon detector, is expected to greatly enhance the physics capability of the $\mathrm{D} \emptyset$ experiment in areas where high resolution tracking with high efficiency is required.

Although the spatial resolution of the tracker can be adjusted by tuning the granularity of

\footnotetext{
"Supported in part by the U.S. Department of Energy, the U.S. National Science Foundation, Korean Ministry of Education, Korean Research Foundation and KOSEF in Korea
}

the fiber, the actual performance of the tracker is directly determined by the light yield of the fiber and the sensitivity of the readout system. Therefore, fibers with the following characteristics have been sought: high light output, large light collection efficiency via total internal reflection, and long attenuation length. The selected fibers must then be coupled to readout devices that provide superior sensitivity at the single photon level. In addition, the fiber should be robust and radiation hard to ensure long term stability during exposure to a large radiation dose.

Polystyrene scintillator using p-terphenyl ( $\mathrm{pT}$ ) and 3-hydroxyflavone (3HF) as the primary and secondary dopants, respectively, is known to be a very efficient scintillator and to have excellent radiation hardness characteristics. In addition, its fluorescence in the wavelength range of 520 to $600 \mathrm{~nm}$ makes it an excellent candidate 
for the core material of a scintillating fiber. Multiclad fiber, fluorinated-PMMA over PMMA over a $\mathrm{pT}+3 \mathrm{HF}$ polystyrene scintillator core, yields a scintillating optical fiber with high light yield[2], excellent attenuation properties, and good radiation stability.

Various fiber readout devices that can be used with a large number of fibers have been studied. Among them, the visible light photon counter(VLPC) [3], has been used by a few groups because of its high quantum efficiency, large gain, low noise, and good stability. Its main drawback is that it has to be operated near liquid helium temperature. The quantum efficiency of this device is known to be more than an order of magnitude higher than that of a conventional photomultiplier in the wavelength range between 520 and $600 \mathrm{~nm}$. The peak sensitivity of the VLPC is well matched to the fluorescence of $\mathrm{pT}+3 \mathrm{HF}$ scintillating fiber $\left(\lambda_{p}=\right.$ $525 \mathrm{~nm}$ ). Earlier test experiments have shown the possibilities of fiber tracking systems using this type of fiber coupled to VLPC readout[2][4][5]. The average number of photons detected in the above experiments was in the range of 6 to 10 photoelectrons.

In the experiment described in this paper, we studied the performance of a 3072 channel scintillating fiber tracker using VLPC readout as a prototype for the central fiber tracker for the upgrade of the $\mathrm{D} \emptyset$ detector. Using data taken with cosmic rays, we studied the system performance of the tracker in terms of optimum bias voltage for the VLPC, tracking resolution, light yield, and efficiency. We also developed various techniques relating to detector fabrication, low temperature control, and bias voltage supply.

\section{EXPERIMENTAL SETUP}

Kuraray multiclad fiber of diameter $835 \mu \mathrm{m}$ [6] was used in the tracker fabrication because of its high light output and good attenuation characteristics. The fiber has a polystyrene core doped with p-terphenyl at a concentration of $1 \%$ by weight and 3-hydroxyflavone at $1500 \mathrm{ppm}$ by weight. The inner and outer claddings are
PMMA and fluorinated-PMMA, respectively,

We formed 128 scintillating fibers of $2.8 \mathrm{~m}$ length into a ribbon, called a "singlet" layer, by laying the fibers into precision grooves in an aluminum jig plate (spacing $870 \mu \mathrm{m}$ ) and then taped them together with a thin $(125 \mu \mathrm{m})$ kapton tape. We then glued two singlet layers together so that one layer was offset by $1 / 2$ a groove spacing relative to the other, thus forming a "doublet."

We formed two super-layers by placing four doublets each on a carbon fiber support cylinder at $90^{\circ}$ and $270^{\circ}$ in $\phi$. The doublets were placed on the surface of the cylinder in the following order; axial, tstereo, -stereo, axial. The stereo doublets have angles of $2^{\circ}$ relative to the axial layers. The carbon fiber cylinder has a radius of $27 \mathrm{~cm}$ and a length of approximately $200 \mathrm{~cm}$. The axial doublet layers run parallel to the cylinder axis. Since these two super layers were mounted on the surface of the cylinder, the ribbons had a curvature. A third super layer was fabricated by mounting 4 doublets on a flat honeycomb carbon fiber board which was then placed in the center of the cylinder. Therefore, the tracker consists of 12 doublet layers or 3072 single fibers in total.

The axis of the cylinder, which is in the horizontal plane, was assigned to the $\mathrm{z}$ direction. The vertical axis which passes through the middle of the cylinder is $y$, and $x$ is defined by a right handed coordinate system. The axial layers measured $\phi$ and the stereo layers measured $z$.

We installed two scintillation counters of about the same area as that covered by the ribbons, just above and below the cylinder. Below the bottom counter were two steel blocks of thickness $0.8 m$ and $1.2 m$, respectively. We installed three additional scintillation counter, one between the steel blocks followed by two that were installed below the second block. The counters were used to form a trigger signal using a multifold coincidence ( 3 or 4 fold) with momentum thresholds of $0.8 \mathrm{GeV} / \mathrm{c}$ or $2.5 \mathrm{GeV} / \mathrm{c}$, respectively. The $r-\phi$ (or $\mathrm{x}-\mathrm{y}$ ) view of the detector setup at one end and the layer configuration are shown in Figure 1 and Figure 2, respectively.

As mentioned above, each singlet ribbon contained 128 fibers. We potted both ends of the fibers in the singlet ribbons into Delrin connectors 


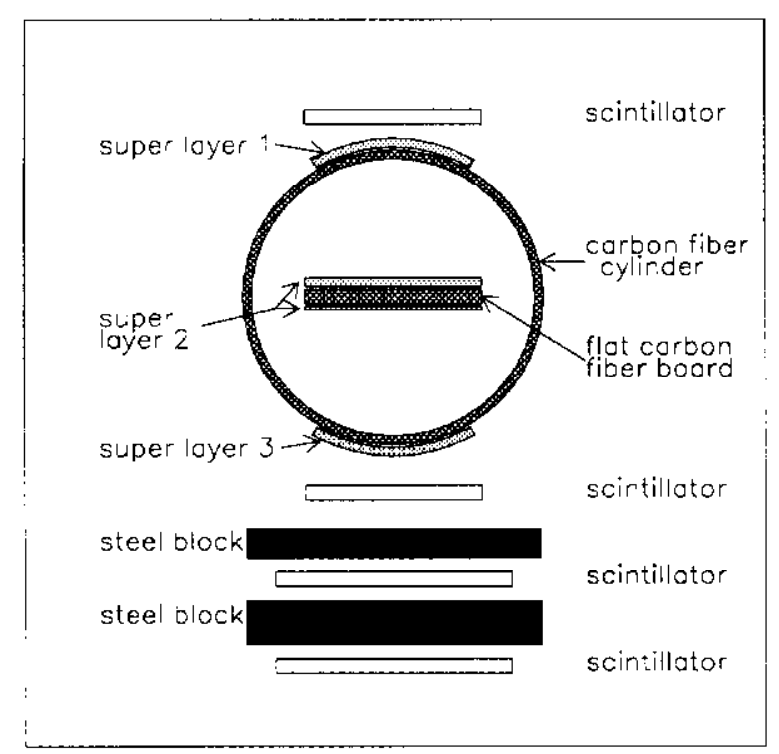

Figure 1. Cross-sectional view of the detector

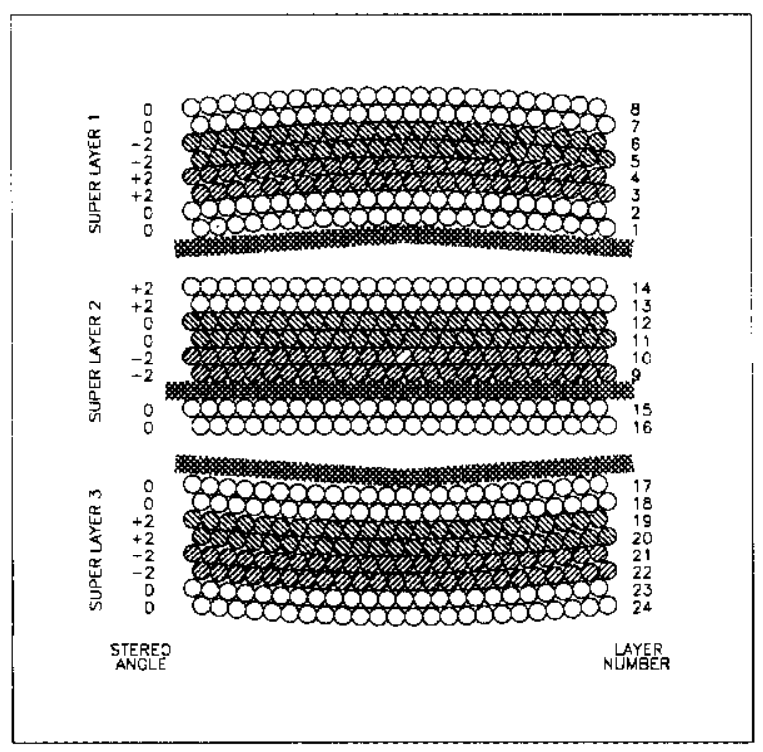

Figure 2. Layer configuration in the super layer. Layer numbering and ordering are shown. using an optical epoxy and then diamond polished each end. We attached the readout end of the ribbon to a connectorized clear fiber bundle (128 fibers of $965 \mu \mathrm{m}$ diameter) of $8 \mathrm{~m}$ length which feeds the light signal to the photodetector. The far end of each fiber ribbon was mirrored with an aluminized mylar sheet and then connected to a $2 m$ long clear fiber bundle that connects each fiber in the system to a LED, so that light could be "injected" into the fibers for calibration and other studies.

The VLPCs are located at the cold end of a cylindrical housing, called a cassette. Each cassette contains 128 channels, enabling it to receive the signal from 128 fibers (one singlet layer). The cassette was inserted into a liquid helium cryogenic vessel and maintained at a temperature of $6.5 \mathrm{~K}$ using a commercial temperature controller. A small heater and a temperature readback sensor were mounted in each cassette. A personal computer provided overall control and data logging for the temperature system. The VLPC bias voltage was controlled by a $\mathrm{PC}$ and supplied from custom VME modules. The preamplifier card for the VLPCs used the Fermilab designed QPA02 [8]. Each cassette used four 32 channel preamp cards.

The HISTE-IV VLPC array contains 8 pixels with a diameter of $1 \mathrm{~mm}$ at a pitch of $1.05 \mathrm{~mm}$. We fully tested and characterized each array before using it in a cassette. The HISTE-IV VLPCs have a typical quantum efficiency of about $60 \%$, a noise rate of $200 \mathrm{kHz}$ (some pixels as high as $1 \mathrm{MHz}$ ) at a $1 / 2$ photoelectron threshold, and a gain of about 15000 at a bias voltage of $6.5 \mathrm{~V}[7]$.

Analog signals from the preamplifiers were sent to Lecroy $1885 \mathrm{~N}$ FASTBUS ADCs and digitized with a gate time of $100 \mathrm{~ns}$. We recorded the data on a $8 \mathrm{~mm}$ tape using a VAXstation II for data acquisition. The trigger rates were approximately $2 \mathrm{~Hz}$ with the $0.8 \mathrm{GeV} / \mathrm{c}$ momentum filter and $0.5 \mathrm{~Hz}$ with $2.5 \mathrm{GeV} / \mathrm{c}$ filter.

\section{TRACKING}

We defined a cluster as a set of three consecutive fibers in a singlet centered on a fiber that had a signal equal to or greater than the 


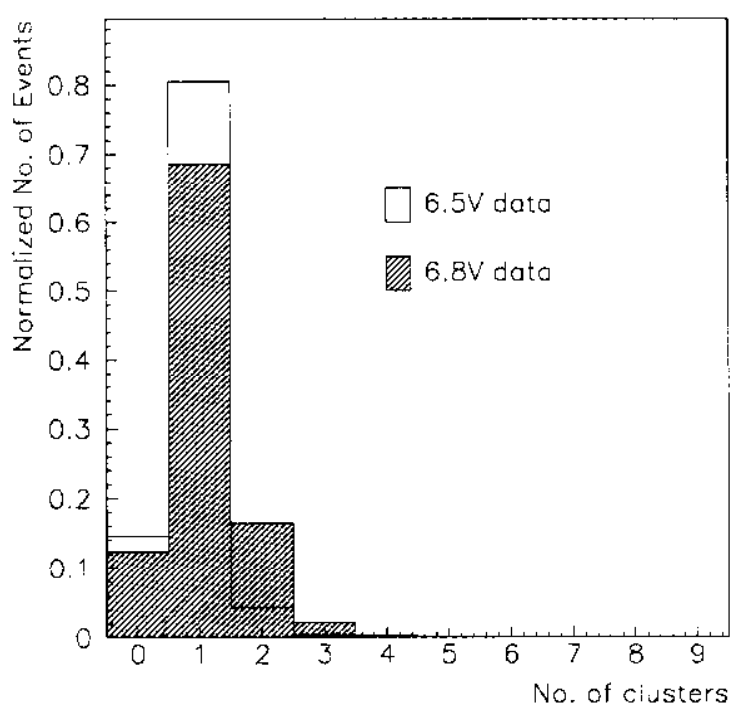

Figure 3. Distribution of the number of clusters at a VLPC bias of $6.5 \mathrm{~V}$ and $6.8 \mathrm{~V}$

pedestal mean $+6 \sigma$. The distribution of the number of the clusters in layer 9 is shown in Figure 3. The average number of clusters at a temperature of $6.5^{\circ} \mathrm{K}$ and at a bias voltage of $6.5 \mathrm{~V}$ was about 0.91 . This number increased to about 1.09 at $6.8 \mathrm{~V}$ due to higher quantum efficiency and higher noise.

In fitting axial tracks, i.e., in the $x-y$ plane, at least 2 singlet layers in each super layer and 7 or more layers in total were required to have a cluster. We fitted a straight line to each possible combination of clusters, using one cluster at a time from each axial layer that had one or more clusters.

In order to remove spurious clusters that might have arisen due to noise, we then compared cluster positions that had been used in the fitting with the expected positions from the fitted line. We removed clusters deviating from the fitted track by more than 0.75 times the fiber spacing and then we again fitted the remaining clusters to a straight line. This procedure was continued until either all the clusters were within the cut or the number of layers which contributed to the track had reached 2 for each super layer or 7 in total. Therefore, some tracks could retain clusters which deviated beyond the cut. We then chose a track with the smallest least square value as a best track.

We fine tuned the cut on the distance between the cluster and the track in order to reject as many spurious clusters as possible while still keeping all real clusters. Theoretically, one can use 0.5 times the fiber spacing for the tightest cut. However, considering misalignment of the fibers, we thought that this value was overly constrained. We used a compromise of 0.75 and this allowed for a misalignment of up to $218 \mu \mathrm{m}$.

To reject tracks that still had redundant hits, and thus would deteriorate track quality, we imposed a mild cut on the final least square value. The tracks surviving this cut were then used for the following analysis.

Once we found the axial track, we used the clusters in the stereo layers to fit a track in the $\mathrm{y}-\mathrm{z}$ plane. The $\mathrm{z}$ tracking used the same number of required layers having clusters as were used in the axial tracking. Given the axial track, we calculated the $\mathrm{z}$ position associated with each cluster on each stereo layer and then we used this position to fit a straight line. Again, we dropped the clusters deviating from the fitted line by more than 0.75 times the projected fiber spacing in the $\mathrm{z}$ direction in the iterations and we used a mild cut on the least square value from the fit to remove tracks with spurious hits.

\section{OPTIMUM BIAS VOLTAGE}

To determine the optimum bias voltage for the VLPCs, we measured a single fiber efficiency at a threshold required to keep the noise rate below a particular value. This efficiency was determined by extrapolating the fitted track into a singlet "test" layer (not used in the fit) and then finding the closest fiber. If the distance of the closest fiber to the track was less than $390 \mu \mathrm{m}$, the active radius of the fiber plus $25 \mu \mathrm{m}$, then the we assumed that the selected fiber was on the track. If the chosen fiber had a signal whose pulse height was above the set threshold, we registered a fiber 


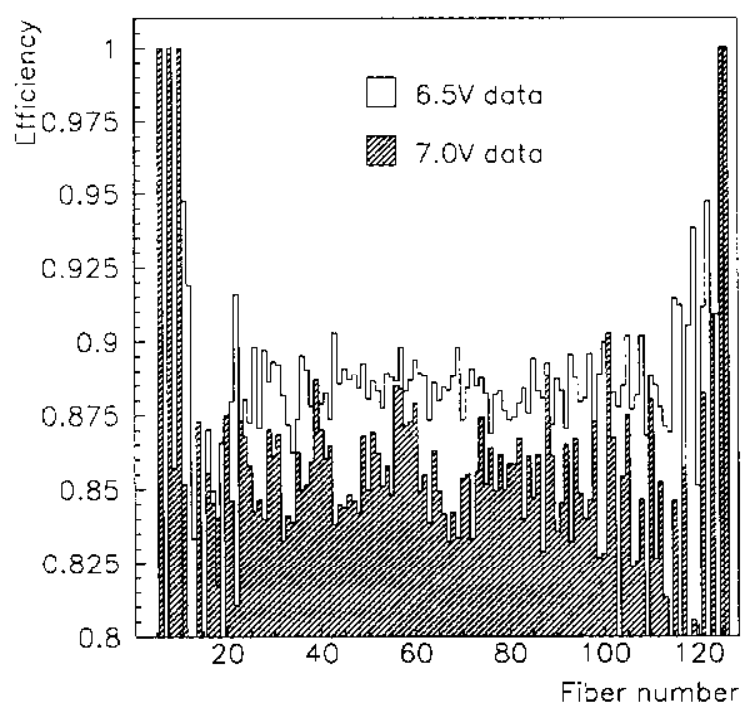

Figure 4. Comparison of single fiber efficiency at $6.5 \mathrm{~V}$ and $7.0 \mathrm{~V}$. Outer fibers suffer from low statistics, but inner ones show that the efficiency at $6.5 \mathrm{~V}$ is slightly better than that at $7.0 \mathrm{~V}$

hit. Fiber by fiber comparisons of the efficiency at $6.5 \mathrm{~V}$ with that at $6.2 \mathrm{~V}, 6.8 \mathrm{~V}$ and $7.0 \mathrm{~V}$ bias voltage showed that the single fiber efficiency, thus determined, varied little in the studied range of bias voltage. However, the efficiency at $6.5 \mathrm{~V}$ appeared marginally favored over the others. In Figure 4, the single fiber efficiencies at $6.5 \mathrm{~V}$ and $7.0 \mathrm{~V}$ are compared. The average value of the single fiber efficiency, measured in this way, was about $88 \%$ at $6.5 \mathrm{~V}$.

\section{RESOLUTION}

The basic technique we used to determine the tracking resolution was to connect two clusters from two different layers that participated in the track fitting. The residual distribution of a cluster from a third layer between the first two layers was then translated into a resolution.

Once we found a track, we connected a cluster in doublet layer 4 (singlet layers 7 and 8 ) with one in doublet layer 9 (singlet layers 17 and 18). We then determined the residual to the track from a cluster in doublet layer 8 (singlet layers 15 and 16). In the case where both of the singlet layers in a doublet layer had a cluster, we used an average position of the two clusters to give a more accurate hit position. The final resolution was then computed by dividing the sigma of the fitted gaussian distribution by $\sqrt{3 / 2}$ in order to take into account contributions to the residual distribution from uncertainties in the cluster positions in the two connected layers. From these fits, we measured the doublet layer resolution in the $r-\phi$ view to be $149.7 \pm 0.5 \mu \mathrm{m}$ with the low momentum filter.

After we rejected low momentum cosmic-ray particles by requiring additional hits from the scintillation counters located under the $2 m$ of steel (the momentum threshold is about $2.5 \mathrm{GeV} / \mathrm{c}$ ), the resolution improved to $136.2 \pm$ $0.6 \mu \mathrm{m}$. Therefore, multiple scattering was a source of resolution deterioration for the low momentum comic-ray cutoff. The residual distribution in the high momentum events is shown in Figure 5.

We calculated the theoretical limit to the resolution to be about $85 \mu \mathrm{m}$ for the double hit case[9] using a simple geometrical Monte Carlo program. This study also showed that the resolution rapidly deteriorated as the misplacement between the singlet layers in a doublet layer increases. We determined the average fiber misplacement in the detector by plotting the difference of the nominal fiber position from the one expected by the track fitting. This measurement yielded a result of approximately $92.5 \mu \mathrm{m}$. At this level of misplacement, the Monte Carlo predicted the resolution to be about $120 \mu \mathrm{m}$. This was comparable to the measured value of $125.5 \pm$ $2.5 \mu \mathrm{m}$ achieved for the double hit case. It seemed that the misplacement between the singlet layers in a doublet layer was a significant component to the overall resolution.

We measured the resolution in the axial (z) direction using the stereo layers in the same way; we connected a cluster in doublet layer 2 (singlet layers 1 and 2) with one in doublet layer 10 (singlet layers 19 and 20) and then we determined 


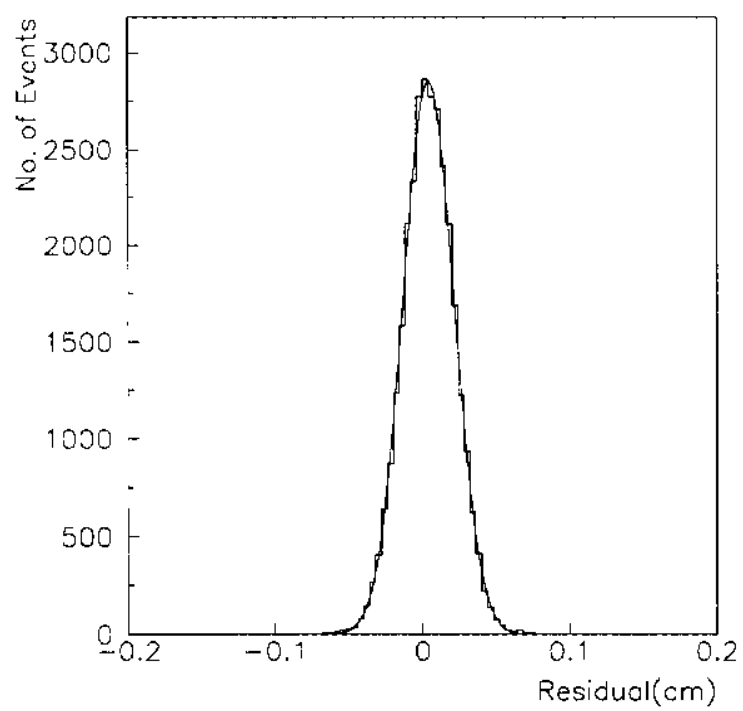

Figure 5. Residual distribution for axial layers. Plotted here is the difference between the position expected by connecting two clusters and the cluster position in the middle layer

Table 1

Doublet resolution

\begin{tabular}{c|c}
\hline momentum & resolution \\
\hline$p>0.7 \mathrm{GeV} / \mathrm{c}$ & $149.7 \pm 0.5 \mu m$ \\
$p>2.5 \mathrm{GeV} / \mathrm{c}$ & $136.2 \pm 0.6 \mu m$ \\
\hline
\end{tabular}

the residual from a hit in doublet layer 7 (singlet layers 13 and 14). The result of $4.17 \pm 0.04 \mathrm{~mm}$ is about 30 times the axial resolution, as expected from the size of the stereo angle. The resolution study for the axial doublet layer is summarized in Table 1.

\section{PHOTOELECTRON YIELD}

To measure the average number of photoelectrons detected by the VLPCs, we probed a "test" doublet layer using tracks fitted without this

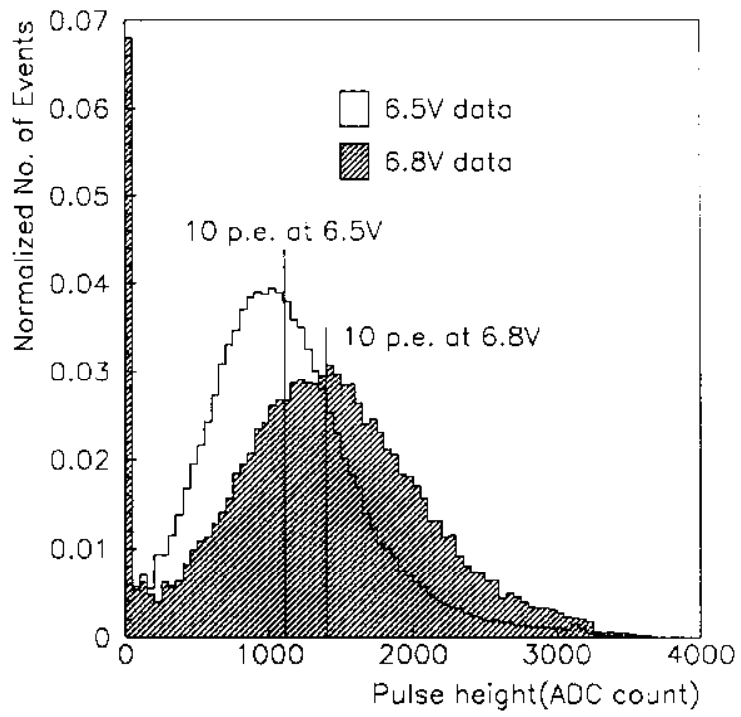

Figure 6. Pulse height spectra of hit fibers in layer 9 at a VLPC bias of $6.5 \mathrm{~V}$ and $6.8 \mathrm{~V}$

layer. We extrapolated the track into the test layer and determined the closest fiber to the track. The pulse height spectra of these fibers, integrated over all $\mathrm{z}$ for VLPC bias voltages of $6.5 \mathrm{~V}$ and $6.8 \mathrm{~V}$, are compared in Figure 6 . We calculated the average number of photoelectrons detected to be about 9.6 at $6.5 \mathrm{~V}$ and 10.0 at $6.8 \mathrm{~V}$ using mean gain values which converted ADC counts into the number of photoelectrons. This is summarized in Table 2 . The gain values were determined by pulsing the fibers with the LED calibration system and identifying the pedestal peak and the first few photoelectron peaks. Typical values for the number of ADC

Table 2

Photoelectron yield(layer 9)

\begin{tabular}{c|c}
\hline Bias voltage & $\left\langle N_{\text {p.e. }}\right\rangle$ \\
\hline $6.5 \mathrm{~V}$ & 9.6 \\
$6.8 \mathrm{~V}$ & 10.0 \\
\hline
\end{tabular}




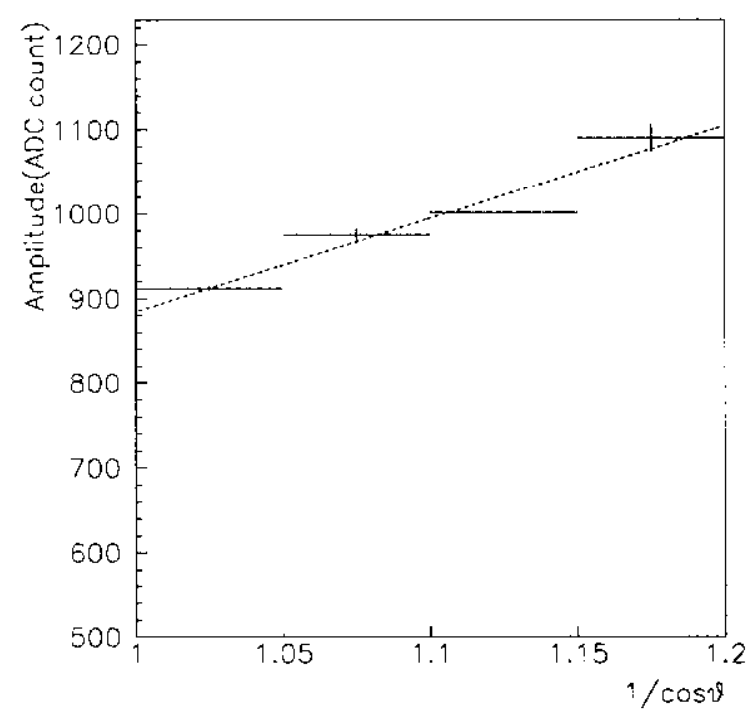

Figure 7. Relationship between peak pulse height position of the hit fibers and $1 / \cos \theta$

counts per photoelectron were 110.8 at $6.5 \mathrm{~V}$ and 139.5 at $6.8 \mathrm{~V}$.

We expected that the light yield would increase with the path length of the particle in the fiber. A measure of the path length is $1 / \cos \theta$ of the track in the $\mathrm{y}-\mathrm{z}$ plane, where $\theta$ is the angle between the track and the $\mathrm{z}$ axis. To confirm whether the light yield increases linearly with $1 / \cos \theta$, we sampled the pulse height spectra in four different $\cos \theta$ bins between 1.00 and 1.20 . We then determined the peak positions of these spectra by fitting a gaussian distribution to the region around the peak. These positions in ADC counts are plotted as a function of $1 / \cos \theta$ in Figure 7. As expected, the light-path length relation is well fitted to a straight line.

To determine the effectiveness of the mirror placed at the far end of the fiber, we took data with and without the mirror and compared the mean photo-yields. Since the intensity of light varies as $I(z)=I_{0} \exp (-z / L)(1+r \exp (-2(L-$ $z) / \lambda$ ) along the fiber where $\lambda$ is the attenuation length of the fiber, $L$ the total length of the fiber, and $r$ the reflectivity, the reflectivity of the mirror could be estimated by comparing the means of the pulse height spectra taken with and without the mirrors. From these measurements, we calculated the reflectivity to be about 0.64 , averaged over all fibers in singlet layers 15 and 16 and with the assumption that $\lambda=500 \mathrm{~cm}$, a value which has been determined from direct attenuation measurements.

\section{EFFICIENCY}

We measured the efficiency of a doublet layer by studying the pulse height of hit fibers in a layer that had not been used in track finding. We determined the hit fibers in the layer by extrapolating the track into the test layer and then taking the two closest fibers to the track. We then determined how often any one of these four fibers (two from each singlet layer, 15 and 16) had a signal above a threshold chosen to keep the noise level below some specified value. We determined five different thresholds by studying pedestal pulse height spectra. The five threshold values were chosen to keep noise rates below $0.025 \%, 0.050 \%, 0.075 \%, 0.100 \%$ and $0.125 \%$, respectively. At a bias voltage of $6.5 \mathrm{~V}$, the mean value of the threshold distribution needed to give a noise rate less than $0.1 \%$ was close to 1 photoelectron. In order to avoid complications due to known hardware problems (bad channels), we discarded an event if any of the closest fibers were flagged for a known problem.

We also estimated the magnitude of the noise contribution to the above efficiency. By looking at uncorrelated events, we were able to determine how often a random hit could contribute to the efficiency, and this was subtracted from the above measured efficiency to result in a net efficiency. The result of this measurement is summarized in Table 3. The doublet layer efficiency was over $100 \%$ even at a threshold corresponding to a $0.025 \%$ noise rate, approximately a 4 photoelectron cut. Since the efficiency saturated even at this rather high threshold, further lowering of the threshold reduced the net efficiency due to increasing noise.

We measured the efficiency of a singlet layer 
Table 3

Doublet efficiency with different noise cuts

\begin{tabular}{c|c}
\hline noise level & net efficiency \\
\hline $0.025 \%$ & $99.60 \pm 0.02 \%$ \\
$0.050 \%$ & $99.53 \pm 0.02 \%$ \\
$0.075 \%$ & $99.44 \pm 0.03 \%$ \\
$0.100 \%$ & $99.33 \pm 0.03 \%$ \\
$0.125 \%$ & $99.25 \pm 0.03 \%$ \\
\hline
\end{tabular}

similarly. The largest pulse height among the two closest fibers to the track in the test layer 15 or 16 was subject to the threshold cut. The singlet layer efficiency measured in this way; was about $85 \%$ at a threshold corresponding to a noise rate of $0.1 \%$ or less.

\section{CONCLUSION}

We measured the system performance of the $D \emptyset$ prototype scintillating fiber tracker using VLPC readout in terms of optimum bias voltage for the VLPC, spatial resolution, light yield and efficiency. The performance of the tracker was well within expectation.

\section{REFERENCES}

1. A. Bross, D $\emptyset$ Upgrade, these proceedings

2. B. Baumbaugh et. al., Performance of multiclad scintillating and clear waveguide fibers read out with visible light photon counters, Nucl. Inst. \& Meth. in Phys. Res. A345(1994)271

3. M.D. Petroff et. al., Detection of individual 0.4-28 $\mu \mathrm{m}$ wavelength photons via impurityimpact ionization in a solid-state photomultiplier, Appl. Phys. Lett. 51(1987)406

4. M. Atac et. al., Tracking with scintillating fibers and visible light photon counters, Nucl. Inst. \& Meth. in Phys. Res. A320(1992)155

5. B. Abott et. al., Beam test of a 12-layer scintillating-fiber charged particle tracking system, Nucl. Inst. \& Meth. in Phys. Res. A339(1994)439
6. Kuraray international corporation, 200 Park Ave., New York, NY 10166

7. S. Grünendahl, Study of a large sample of visible light photon counters, these proceedings

8. T. Zimmerman, A high speed, low noise ASIC preamplifier for silicon strip detectors, IEEE Trans. on Nucl. Sci. 37(1990)439

9. D. Koltick, The effect of scintillating fiber layer misalignment on doublet resolution, unpublished 